\title{
Fluid Biomarkers for Mild Traumatic Brain Injury and Related Conditions
}

Henrik Zetterberg ${ }^{1,2,3}$ and Kaj Blennow ${ }^{1,2}$

${ }^{1}$ Department of Psychiatry and Neurochemistry, Institute of Neuroscience and Physiology, the Sahlgrenska Academy at the University of Gothenburg, Mölndal, Sweden;

${ }^{2}$ Clinical Neurochemistry Laboratory, Sahlgrenska University Hospital, Mölndal, Sweden

${ }^{3}$ Department of Molecular Neuroscience, UCL Institute of Neurology, Queen Square, London, $U K$

Correspondence:

Henrik Zetterberg, $M D, P h D$

Professor of Neurochemistry

Head of Department

Department of Psychiatry and Neurochemistry

Institute of Neuroscience and Physiology

The Sahlgrenska Academy at the University of Gothenburg

S-431 80 Mölndal

SWEDEN

Tel (office): +46313430142

Tel (cell): +46 768672647

Tel (secretary): +46 313430025

Fax: +4631 419289

E-mail: henrik.zetterberg@gu.se 
Kaj Blennow, $M D, P h D$

Professor of Neurochemistry

Department of Psychiatry and Neurochemistry

Institute of Neuroscience and Physiology

The Sahlgrenska Academy at the University of Gothenburg

S-431 80 Mölndal

SWEDEN

Tel (office): +46313431792

Tel (cell): +46 761073835

Tel (secretary): +46313430025

Fax: +4631419289

E-mail: kaj.blennow@neuro.gu.se 


\begin{abstract}
Moderate to severe traumatic brain injury (TBI), where there is gross damage to the brain parenchyma, may be diagnosed by clinical assessment and standard neuroimaging techniques. In contrast, diagnostic tools for mild TBI or concussion are lacking; currently, the diagnosis has to be made on clinical grounds. Most mild TBI patients recover quickly but a proportion develop an ill-defined condition called post-concussive syndrome (PCS). Repetitive concussions have also been associated with a chronic neurodegenerative disorder called chronic traumatic encephalopathy (CTE), which is a diagnosis that only can be made post mortem. There is an unmet clinical need of diagnostic and prognostic biomarkers for mild TBI. We also need research tools to better define PCS and to detect CTE in vivo. Here, we review the literature on fluid biomarkers for neuronal, axonal, oligodendrocytic, astroglial and blood-brain barrier injury, as well as neuroinflammation and metabolic dysregulation, in the context of mild TBI and related conditions. We also discuss technical and standardisation issues and potential pathways forwards regarding how to move the most promising biomarker candidates into clinical laboratory practice.
\end{abstract}




\section{Key points:}

- Traumatic brain injury is caused by an external mechanical force injuring the brain parenchyma.

- Repetitive mild traumatic brain injury may cause long-term symptoms and progressive neurodegeneration, but how big this risk is, is unknown.

- Mild traumatic brain injury is hard to diagnose objectively; accurate fluid biomarkers would be a welcome addition to the diagnostic toolbox.

- Fluid biomarkers for axonal injury and astroglial activation show the greatest promise at the moment but several other promising biomarker candidates exist.

- Brain specificity or brain-enhanced expression is an important characteristic of bloodbased biomarkers for mild traumatic brain injury, as extra-cerebral sources otherwise may compromise the interpretability of the test results. 


\section{Biographies}

Henrik Zetterberg, MD, PhD, is a Professor of Neurochemistry at the University of Gothenburg, Sweden, and University College London, UK, and a Clinical Chemist at the Sahlgrenska University Hospital in Gothenburg, Sweden. He is Head of the Department of Psychiatry and Neurochemistry at the University of Gothenburg and his main research focus and clinical interests are fluid biomarkers for central nervous system disorders.

Kaj Blennow, MD, $\mathrm{PhD}$, is Head of the Clinical Neurochemistry Laboratory at Sahlgrenska University Hospital and Professor of Clinical Neurochemistry at the University of Gothenburg, Sweden. His main research interests are fluid biomarkers for Alzheimer's disease and other brain disorders, and the neurochemical pathogenesis of Alzheimer's disease and traumatic brain injury. He has received numerous prizes and awards, most recently the Torsten Söderberg Prize of Medicine. 


\section{Review criteria:}

We searched PubMed for articles in the English language on mild traumatic brain injury (TBI) and related conditions using the keywords "TBI" or "traumatic brain injury" or "concussion", in combination with "biomarkers", "CSF", "plasma", "serum", "blood", "mild traumatic brain injury", "mild TBI”, "post-concussive/concussion syndrome”, "PCS”, "chronic traumatic encephalopathy" or "CTE". We selected articles examining fluid biomarker changes in mild TBI, PCS and CTE. We also included articles examining biomarkers in moderate to severe TBI, when of relevance to mild TBI, PCS and/or CTE. 


\section{Introduction}

Traumatic brain injury (TBI) is caused by an external mechanical force impacting the brain. Moderate to severe TBI, in which there are intracranial bleedings and/or mass lesions in the brain parenchyma, is diagnosed by clinical examination and standard neuroimaging techniques, such as computed tomography $(\mathrm{CT})$ or magnetic resonance imaging (MRI) of the brain. ${ }^{1}$ Clinically, TBI may also be graded using the Glasgow Coma Scale, in which the patient's conscious state is rated on the basis of eye, verbal and motor responses to different stimuli. ${ }^{2}$ However, the prognostic utility of this and similar scales is under debate and additional sub-classification with novel biomarkers remains an unmet clinical need, as extensively discussed elsewhere. ${ }^{3}$

Mild TBI or concussion (the terms are synonymous) is defined as a head trauma resulting in brief loss of consciousness and/or alteration of mental state (Box 1), which is hard to objectively diagnose. Today, this is a clinical diagnosis based on self-reported symptoms after blunt head injury and presents an everyday challenge in emergency care units around the world. There are several reasons for why the diagnosis should not be neglected. First, approximately $7-20 \%$ of consecutive patients presenting to the emergency room with suspected concussion have an intra-cranial abnormality (e.g., contusion or haemorrhage) on a day-of-injury CT scan. ${ }^{4}$ Second, a significant number of mild TBI patients get incapacitating symptoms for $>10$ days following the injury, which is often termed post-concussive syndrome (PCS). ${ }^{5}$ Third, reports linking chronic traumatic encephalopathy (CTE) with repetitive mild TBI suggest that care should be taken to minimise the risk of a new concussion before proper recovery has been secured. ${ }^{5}$ 
Concussion/mild TBI per se causes no gross pathology, such as haemorrhage, and no abnormalities on conventional CT scans of the brain, ${ }^{6}$ but rather rapid-onset neuronal dysfunction that often resolves in a spontaneous manner over a few days to weeks. The major cause of the symptoms in mild TBI is thought to be axonal injury caused by rapid stretching of the axons due to acceleration/deceleration and/or rotational forces on the brain parenchyma. ${ }^{5}$ Neuroinflammation/microglial activation may also play a role. Novel MRI techniques, e.g., diffusion tensor imaging (DTI), may be used to monitor axonal integrity, but conclusive evidence on their sensitivity to detect axonal injury in mild TBI is lacking. ${ }^{7,8}$

Approximately $15 \%$ of concussion patients suffer cognitive or neurological dysfunction that continues for months or more after the initial injury. ${ }^{9,10}$ Such patients may fulfil criteria for PCS. ${ }^{11}$ PCS has been the subject of intense debate, since the diagnosis is associated with quite a wide range of symptoms (physical, such as headache; cognitive, such as difficulty concentrating; and emotional/behavioural, such as irritability), and since the risk of PCS is influenced not only by injury mechanisms but also by psychological and socioeconomic factors. ${ }^{12}$ It is not known what causes PCS to occur and persist. ${ }^{12-14}$

Repetitive concussions are thought to be a risk factor for CTE, a neuropathological diagnosis that was first described in boxers, ${ }^{15}$ but more recently also in other sports athletes, as well as in military veterans. ${ }^{16}$ Clinically, the condition is characterized by chronic and sometimes progressive neurological and/or psychiatric symptoms. ${ }^{16}$ The molecular pathology of CTE is characterized by tau-positive neurofibrillary tangles and neuropil threads that may be present in all regions of the brain. ${ }^{16}$ Neurofibrillary tangles in CTE form preferentially in the superficial cortical layers, rather than in deeper layers as is more common in Alzheimer's disease, with focal accumulations at the depths of the sulci. ${ }^{17}$ Another distinctive (perhaps the 
most distinctive) feature of CTE is perivascular tau deposition, which may reflect an association of tau deposition with TBI-related injury to cerebrovascular endothelial cells, which by unknown mechanisms may promote tau deposition. ${ }^{17}$ The relation between PCS and future development of CTE is unknown. Potentially, a fraction of PCS patients will eventually be diagnosed with CTE; the size of this fraction is currently unknown given the lack of in vivo tools to diagnose CTE in living humans.

\section{Biomarkers}

A fluid biomarker is defined as a molecule that is measureable (as a concentration or an activity, or as a qualitatively distinct form of the molecule) in a biological fluid for which the measure relates to a physiological or pathological process occurring in a living cell or organism. In TBI research and clinical work-up, fluid biomarkers have the potential to help to define: (i) TBI severity (including the severity of different types of injury, e.g., axonal injury and microvascular damage); (ii) adaptive and recovery processes following TBI; (iii) the transition between a normal injury/recovery pattern and a progressive CTE process; and (iv) treatment response in clinical trials. For most of the biomarker candidates discussed below these potential contexts of use have not yet been defined in appropriate clinical settings.

\section{Sample types for fluid biomarker research in TBI}

\section{Cerebrospinal fluid}

Cerebrospinal fluid (CSF) is a clear fluid that surrounds the brain and provides mechanical support. It also carries nutrients and signalling molecules to neurons and helps disposing metabolites that are further cleared into the blood via arachnoid villi in the intracranial dural sinuses and at the cranial and spinal nerve root sheaths, ${ }^{18}$ as well as through meningeal lymphatic vessels. ${ }^{19}$ The recent discovery of the so-called glymphatic system has helped 
explaining how subarachnoid CSF may enter and exit the brain along paravascular spaces and clear the brain parenchyma from extracellular metabolites and other breakdown products. ${ }^{20}$ The total CSF volume is around $150 \mathrm{~mL}$ and the production and clearance rate is around 20 $\mathrm{mL}$ per hour. Standard operating procedures for CSF sampling via lumbar puncture have been established and the procedure can be performed in outpatients. ${ }^{21}$ Lumbar puncture is safe with post-lumbar puncture headache being the only significant side-effect that affects $2-20 \%$ of the

patients. $^{22}$ In severe TBI, CSF may be sampled through intra- or extraventricular catheters but it is important to take into account that intracranial CSF differs in protein composition from lumbar CSF, so that biomarker concentrations from the two fluid types cannot be compared directly. ${ }^{23}$ The main advantage of CSF as a matrix in which to measure markers of CNS injury is that it communicates freely with the brain interstitial fluid that bathes the neurons.

Biochemical changes in the brain are thus often reflected in the CSF, which may be regarded as an accessible, although not perfect, sample of the brain interstitial fluid. Further, CSF has low protease activity and most molecules do not change upon sampling provided the sample is not contaminated by blood. The main disadvantage is that lumbar puncture may be regarded as invasive. Finally, although CSF allows sampling from the brain side of the blood-brain barrier, it should be remembered that only $20-30 \%$ of the CSF volume is derived from the brain; $70-80 \%$ is a filtrate of plasma, produced by the choroid plexus. ${ }^{18}$

\section{Blood}

The other major biofluid for TBI biomarker analysis is blood (serum or plasma), which communicates with the brain and CSF compartments through the glymphatic system. ${ }^{20}$ There are several issues, both biological and technical, with the measurement of CNS-related biomarkers in blood. First, a biomarker that has its origin in the CNS has to cross the bloodbrain barrier in order to be detected in the periphery and, if the concentration is low in CSF, it 
will be even lower in the blood due to the blood:CSF volume ratio causing a substantial dilution. Second, if the biomarker is not specific for the CNS but also expressed in peripheral tissues that may be injured in trauma, the contribution from CNS will potentially drown in the high biological background caused by non-CNS sources (a good tool to assess the risk for this is the publicly available web-based Human Protein Atlas, http://www.proteinatlas.org/, which presents mRNA and protein expression in 44 different human tissues of close to 20000 proteins).$^{24}$ Third, the huge amount of other proteins in blood (e.g., albumin and immunoglobulins) introduces analytical challenges due to possible interference. ${ }^{25}$ Fourth, heterophilic antibodies may be present in blood, which may interfere in immunoassays (see below for more details). ${ }^{26}$ Fifth, the analyte of interest may undergo proteolytic degradation by various proteases in plasma. ${ }^{27}$ Sixth, clearance of the biomarker in the liver or by the kidneys may introduce variability. Finally, recent data, derived from a mouse model of TBI, suggest that sleep deprivation and cisternotomy may inhibit glymphatic clearance and thereby suppress TBI-induced increases in serum biomarkers. ${ }^{28}$ Whether this potential confounder is relevant also in humans remains to be examined.

\section{Saliva, urine and tears}

It is possible that some CNS-derived proteins are eventually excreted into body fluids other than CSF and blood. The presence of the CNS-enriched protein tau in saliva has been demonstrated using mass spectrometry. ${ }^{29}$ The same research group has also detected Parkinson-related $\alpha$-synuclein and DJ-1 in this body fluid. ${ }^{30}$ However, the relationship between salivary concentrations of these proteins to processes within the CNS is far from clear and no conclusive data on disease association has been reported so far. At present, it is hard to imagine how a test based on sampling of saliva, urine or tears could produce results with a clear link to changes in the brain, given the many barriers and compartments the 
marker has to cross on its way to the sampling site. Further, expression of, e.g., tau mRNA and protein in both salivary glands and kidney

(http://www.proteinatlas.org/ENSG00000186868-MAPT/tissue), may limit the interpretability of measurements in these fluids further. Since these fluids, in our opinion, are unlikely to yield useful markers, they will not be discussed further.

\section{Measurement techniques}

Most biomarker assays of relevance to TBI are immunochemical, i.e., utilise antibodies to quantify a substance in a sample. The most common assay format is the sandwich enzymelinked immunosorbent assay (ELISA) in which the target analyte is captured between two antibodies in a complex and one of the antibodies carries a signal generator, i.e., an enzyme that converts a substrate into a detectable form (coloured, fluorescent or luminescent), which, in combination with a calibrator curve (derived from artificial samples with known analyte concentrations), allows for quantification of the analyte of interest. ELISA is a theme with many variations, such as the choice of signal generator where the enzyme can be replaced by, e.g., a fluorophore or a DNA-based detection system.

Immunochemical assays may also be multiplexed in different ELISA-like formats to allow for the simultaneous measurement of markers reflecting distinct and unique axes of the pathobiology of TBI in the same sample. Such biomarker panels are frequently examined in the current biomarker literature with focus on CNS disorders, ${ }^{31}$ and could potentially provide more comprehensive and complementary information and permit a more accurate characterization and risk stratification of patients with TBI. A downside with multiplexing, however, is that it may be hard to optimize the analytical conditions for several antigenantibody interactions, especially if their concentrations or biochemical characteristics 
substantially differ, as compared to optimizing an assay that focuses on the accurate measurement of a single analyte.

The technical issues are mainly a question of antibody sensitivity and specificity. In theory, if the time for the enzyme reaction is simply extended, this should increase the sensitivity of the assay. However, the substrates used are inherently unstable and therefore produce signal even in the absence of enzyme. This leads to a technical background signal that can mask the signal generated by the sandwich complex, making quantification difficult at low concentrations. In the end, the ability of the sandwich complex to correctly represent the concentration of the biomarker in a sample strongly depends on the quality of the antibodies used. If the antibodies cross-react with other substances, a signal can be measured even in the absence of the target analyte. Since the blood is much denser in protein content than is CSF, the risk for this is higher in the former, where even minor (e.g., $0.1 \%)$ cross-reactivity against proteins present at one million times higher concentrations will have a large impact on the measured concentration.

For the problem with background, new ultrasensitive technologies provide a number of solutions. Most of these technologies rely on antibody-based detection of the target molecule, but in Single molecule array (Simoa), the detection reaction is compartmentalized into a small volume (50 femtolitres), so that the reporter molecule accumulates at a very high concentration, ${ }^{32}$ in Single molecule counting (Singulex), the labelled detection antibodies, specifically captured by the target molecule/capture antibody complex, are released and counted one by one in a small detection cell, which allows for a single molecule read-out, ${ }^{33}$ and in proximity extension assay (PEA), partly overlapping complementary DNA strands are attached to the different antibodies allowing the strands to form a polymerase chain reaction- 
amplifiable template if immobilised close to each other on the same molecule. ${ }^{34}$ These variations in signal generation/detection may result in assays that can be 10 - to a 1000 -fold as sensitive as the corresponding regular ELISA using the same antibody pair.

When using antibody-based assays on plasma or serum samples, it is essential to evaluate the assay for potential interference of human anti-mouse antibodies (HAMAs). These are heterophilic antibodies that can bind mouse (or other species) antibodies and bridge the capture and detection antibodies, thereby replacing the analyte and giving a falsely high signal. $^{26}$ The prevalence in humans of such antibodies may be around $5 \% .{ }^{35}$ There are several commercially available blockers against HAMAs; most are simply based on the addition of an excess of polyclonal mouse $\operatorname{IgG}$ that would sequester any interfering antibodies.

Mass spectrometry (MS)-based assays are increasingly important in clinical laboratory medicine, mostly to measure small molecules, such as drugs, amino acids, hormones and

vitamins in an antibody-independent manner. ${ }^{36}$ Mass spectrometers are also used in explorative proteomics studies to identify new biomarker candidates. However, explorative proteomics has so far failed to generate validated TBI biomarkers and, in general, MS-based standardised quantification of peptides and proteins for routine diagnostic use remains rare. ${ }^{37}$ There are so far no established MS-based assays for TBI biomarkers, but this may well change during the coming years thanks to rapid technical development.

\section{Fluid biomarkers for acute TBI}

A schematic representation of established and candidate fluid biomarkers for neuronal, axonal, oligodendrocytic, astroglial and blood-brain barrier injury, as well as 
neuroinflammation, in TBI is given in Figure 1. Since TBI is a condition with a distinct onset and since there are different phases of pathophysiological responses post-TBI, ${ }^{38}$ the timing of the biomarker sampling is crucial. If the intended use of the biomarker candidate is to quantify the initial damage, such a biomarker should probably be examined as early as possible, taking biomarker kinetics into account, whilst a biomarker for a delayed response, e.g., neuroinflammation or Wallerian degeneration, may be negative at early time points and reach its maximum concentration days, or even weeks to months, following the trauma (Figure 2). Below, we give a detailed account of the biomarker candidates for acute TBI that have been examined so far.

\section{Neuronal injury}

Neuron-specific enolase (NSE) is a glycolytic enzyme that converts 2-phosphoglycerate to phosphoenolpyruvate. ${ }^{39}$ The protein is enriched in neuronal cell bodies, ${ }^{40}$ but is also found in neuroendocrine cells, oligodendrocytes, blood platelets, and at particularly high concentrations in erythrocytes. ${ }^{39,41}$ This is the major limitation of the maker; also invisible haemolysis will contaminate the sample. ${ }^{42}$

NSE concentrations are higher in ventricular CSF from non-survivors compared with survivors hours to days following severe TBI and correlate with other TBI severity scores in both adults and children. ${ }^{43-47}$ To what extent these findings depend on release of NSE from injured neurons or lysed erythrocytes is currently unknown; studies in which NSE concentration is related to free haemoglobin are needed.

Numerous studies have examined NSE in serum as a potential biomarker for mild TBI. Initial results were promising showing increased serum NSE concentrations in samples taken from 
patients with mild TBI within a few hours post-injury, ${ }^{48-50}$ but when scrutinizing the data, the overlap with non-concussed controls makes the diagnostic utility uncertain. One study examined the diagnostic performance of serum NSE concentration at 3 hours post-injury to identify intracranial lesions in mild TBI and found a sensitivity and specificity of $56 \%$ and $77 \%$, respectively. ${ }^{51}$ A recent study on sports-related concussion also produced disappointing results with no increase in serum NSE concentration over hours to days following concussion compared with preseason concentrations. ${ }^{52}$ Altogether, serum NSE does not appear to be a sensitive enough blood biomarker for mild TBI.

Another protein enriched in the cytoplasm of neurons and a candidate biomarker for TBI is ubiquitin C-terminal hydrolase-L1 (UCHL1). However, UCHL1 is also expressed in neurons of the peripheral nervous system, ${ }^{53}$ as well as in cells of the neuroendocrine system, ${ }^{54}$ endothelial and smooth muscle cells, ${ }^{55}$ and in some tumours. ${ }^{56}$ Nevertheless, increased serum and CSF concentrations of UCHL1 have been reported in patients with severe TBI with the highest concentrations seen within 24 hours post-injury in patients with poor outcome. ${ }^{57}$ Quite a number of papers have examined serum UCHL1 as a potential biomarker for mild TBI but the studies have been small and the results inconsistent. ${ }^{58}$ In one of the larger studies, UCHL1 concentrations measured in serum samples collected within 4 hours post-injury from patients with mild ( $n=86)$ and moderate $(n=10)$ TBI, as well as from trauma and non-trauma controls, were increased in both TBI groups compared with the control groups. ${ }^{59}$ Another study reported that serum UCHL1, taken within 24 hours post-injury, could distinguish mild TBI patients with intracranial lesions from those without intracranial lesions with an area under the curve of $0.713,{ }^{60}$ a result that calls for additional replication studies. 
Yet another protein with enhanced expression in neurons, as compared to other tissues, is brain-derived neurotrophic factor (BDNF). This protein is a member of the nerve growth factor family and activation of its intracellular receptors may produce neural regeneration, reconnection, and dendritic sprouting. ${ }^{61}$ One could imagine that neuronal injury in TBI would result in increased fluid concentrations of this potential biomarker but instead it appears like insufficient release of the protein may be related to poor outcome; in a recent study, day-ofinjury serum BDNF concentrations were lower in TBI patients compared with controls, particularly so in cases with severe TBI. ${ }^{62}$ However, another recent study performed in plasma did not support an association of BDNF concentration with outcome. ${ }^{63}$

\section{Axonal injury}

The best-established CSF biomarkers for axonal injury are tau and neurofilament light (NF-L) and heavy (NF-H). ${ }^{64}$ Tau is a microtubule-associated protein abundant in axons, but can also be detected in liver, kidney and testis. ${ }^{65}$ Further, a larger isoform of tau (called "big tau"), containing the epitopes used to measure tau with the currently available immunoassays, is expressed in peripheral nerves. ${ }^{66}$ Phosphorylation of tau is a physiological phenomenon but hyper-phosphorylation and aggregation into neurofibrillary tangles is characteristic of Alzheimer's disease and CTE. ${ }^{65}$ In CSF, it is possible to measure both total tau (T-tau; with assays that do not discriminate between unphosphorylated and phosphorylated forms of tau, or between tau molecules translated from differently spliced mRNAs) and phosphorylated tau (P-tau; with sandwich immunoassays in which at least one of the antibodies is specific to a phosphorylated epitope of tau).

NF-L and NF-H are also structural axonal proteins but, in contrast to tau that is predominantly expressed in thin unmyelinated axons of the cortex, ${ }^{67}$ the expression of neurofilaments is high 
in large-calibre myelinated axons that extend subcortically. ${ }^{68}$ Expression is also detected in peripheral nerves. ${ }^{68}$

T-tau concentrations in ventricular CSF correlate with lesion size and outcome in severe TBI, so that high levels indicate worse injury. ${ }^{69-71}$ In contrast, CSF P-tau concentration, as measured by currently available P-tau assays, is unchanged in TBI and other forms of acute brain injury. ${ }^{72,73}$ Studies on mild TBI show increased CSF concentrations of both T-tau and NF-L, but the magnitude of the increase of CSF NF-L is greater than for T-tau, suggesting that blows to the head impact long, large-calibre axons that extend subcortically more than short, non-myelinated axons in the cortex. ${ }^{73,74}$ Similar results as for NF-L have been observed for CSF NF-H. ${ }^{75}$

Serum tau concentrations have been examined in both paediatric and adult patients with mild TBI and related to findings on CT scans of the brain. Concussed patients showed increased serum tau concentrations as compared to non-concussed controls, ${ }^{76,77}$ but there was no difference between patients with a positive or negative CT scan. ${ }^{76,78}$ However, in these studies, a regular tau ELISA, not optimised for the low concentrations of tau in the blood and not containing blockers against heterophilic antibodies in the sample diluent, was used, making the data uncertain. In moderate and severe TBI, requiring treatment at a neurointensive care unit, serum NF-L concentration measured by regular ELISA was increased, but no correlation with DAI or intracranial damage assessed by CT was seen. ${ }^{79}$

Standard ELISAs for T-tau and NF-L were recently transferred onto the Single molecule array (Simoa) platform, which allows for the ultrasensitive measurement of these two proteins in the blood. ${ }^{80,81}$ Plasma T-tau concentration correlates poorly with CSF concentration, ${ }^{82}$ but in 
acute hypoxic brain injury, a biphasic release of tau into the bloodstream was observed with a first peak occurring during the first few hours post-injury and a second broad peak occurring after a few more days; these increases were predictive of outcome ${ }^{80}$ In a study on concussed professional ice hockey players, plasma T-tau concentration at 1 hour post-injury was increased compared with preseason concentrations and predicted return-to-play time with high accuracy. ${ }^{52}$ In another study of 34 patients with varying degree of TBI, plasma tau concentration in samples collected within 24 hours post-injury showed high accuracy to differentiate complicated mild TBI from controls (area under the curve 0.901). ${ }^{83}$ Recently, Olivera et al. reported increased mean plasma tau concentration in military personnel with a history of self-reported TBI compared with controls without such a history. ${ }^{84}$ Additionally, post-concussive symptoms correlated with plasma tau concentration in the TBI group, suggesting that the increase, although variable, might be of pathophysiological relevance. ${ }^{84}$

Tau can be proteolytically cleaved by activated caspases and calpains in axonal injury, which generates fragments that can be measured in blood. Serum tau fragment concentrations in concussed ice hockey players were variable but peaked at 12 hours post-injury with the highest concentrations seen in those for whom it took longer than 10 days to become asymptomatic. ${ }^{85}$ Serum tau fragments did not correlate with post-concussive symptoms at three months following mild TBI, suggesting that it is not a late marker. ${ }^{86,87}$

Serum NF-L concentration measured by Simoa correlated strongly with CSF NF-L, ${ }^{81,88}$ and increased over time in American football athletes over the course of a season, ${ }^{89}$ which may reflect head impacts, although it should be noted that changes were minor and that no direct assessment in relation to concussion has been performed to date. Gatson et al. examined serum concentrations of NF-H in mild TBI days 1 and 3 post-injury ${ }^{90}$. At both time points, 
serum NF-H concentrations were increased compared with concentrations in control individuals. Serum NF-H concentration was higher in CT-positive compared with CTnegative patients, and was inversely correlated with clinical severity scores, ${ }^{90}$ which is a promising result in need of replication.

The contribution of extra-cerebral sources of tau and neurofilaments to the results above is currently unknown and has to be carefully examined in future studies. However, for tau, plasma concentrations did not increase upon participation in an ice hockey game without incident concussions. ${ }^{52}$ These results contrast, e.g., S-100B and suggest that the brainenhanced expression of tau is high enough to make blood data interpretable in concussion. ${ }^{52}$

$\alpha I I-S p e c t r i n ~ b r e a k d o w n$ products (SBDPs) have been described as potential biomarkers for brain injury in rats and humans. ${ }^{91,92} \alpha \mathrm{II}-$ Spectrin is abundant in axons and presynaptic terminals. In TBI and other forms of acute neuronal injury, the protein is processed to breakdown products by calpain and caspase- 3 that are activated in dying neurons. SBDPs have been measured in ventricular CSF from patients with severe TBI and the concentrations correlate with clinical measures of TBI severity and outcome. ${ }^{93-95}$ In plasma, SBDP concentration increased within 24 hours after severe TBI. ${ }^{96}$ In mild TBI, also in some CTnegative cases, serum SBDP concentration was also increased. ${ }^{97}$ Similar results were recently obtained in sports-related concussion (increased serum concentrations of SBDP 1 hour up to 6 days post-injury). ${ }^{98}$

\section{Oligodendrocytic injury}

Myelin basic protein (MBP) is a major component of the myelin sheath made by oligodendrocytes of the central nervous system (CNS) and Schwann cells of the peripheral 
nervous system. ${ }^{99} \mathrm{MBP}$ is found at increased concentrations in the CSF of patients with demyelinating diseases such as multiple sclerosis. ${ }^{100}$ Since myelinated axons are thought to be primary targets in DAI, MBP has been suggested as a potential biomarker for TBI. The literature so far on the topic is scarce but MBP concentrations in CSF collected from extraventricular drains in severe paediatric TBI were found to be manifold increased as compared to CSF MBP concentrations in lumbar CSF from children evaluated for suspected CNS infection (the normal difference between interstitial fluid MBP and lumbar CSF MBP concentrations is unknown, however). ${ }^{101}$ Further, serum MBP concentration may be increased in paediatric TBI with intracranial haemorrhage compared with mild TBI without haemorrhage. ${ }^{102}$ Finally, increased serum MBP concentration has been detected in adult TBI patients with secondary brain hypoxia. ${ }^{103}$ Data from one study suggests that serum MBP concentration may peak later than S-100B and NSE; between $48 \mathrm{~h}$ and $72 \mathrm{~h}$ post-injury. ${ }^{102}$ MBP may therefore have the greatest potential in post-acute mild TBI.

\section{Astroglial injury}

S-100B, an astrocyte-enriched $\mathrm{Ca}^{2+}$-binding protein, has been examined extensively in relation to TBI in serum, but studies on CSF are rare. Amateur boxers have slightly elevated CSF levels after bout, but the changes are not as pronounced as those for the axonal markers T-tau and NF-L. ${ }^{73}$ Similar results have been reported for glial fibrillary acidic protein (GFAP), ${ }^{73,74}$ which is an intermediate filament that is almost exclusively expressed in astrocytes. ${ }^{40}$ However, CSF GFAP has also been evaluated in the context if severe TBI and ventricular levels may improve outcome prediction models in conjunction with clinical data. ${ }^{95}$ Clinically relevant changes in serum concentrations of S-100B and GFAP have been reported to detect radiographically apparent intracranial injury. ${ }^{104-106}$ The data from one of these studies suggested a slightly higher diagnostic accuracy for GFAP compared with S-100B, 
particularly in patients with extracranial fractures that may give increased serum levels of S100B due to release of the protein from bone. ${ }^{107} \mathrm{~S}-100 \mathrm{~B}$ has been included as a biomarker that could reduce the number of unnecessary CT scans of the brain in new clinical guidelines for the management of head injury. ${ }^{104}$ Still, this remains a controversial field; in a large convenience sample of adult trauma patients, the area under the curve of S-100B for detecting intracranial lesions on CT using serum samples obtained within 4 hours post-injury was only $0.78(95 \%$ confidence interval $0.67-0.89) .{ }^{106} \mathrm{~A}$ recent study of 34 patients with varying degree of TBI showed excellent accuracy of plasma GFAP to differentiate complicated mild TBI from controls (area under the curve 0.936 for samples collected during the first day post$\mathrm{TBI})^{83}$

\section{Blood-brain barrier function}

Evidence from experimental and human autopsy studies indicate that blood-brain barrier disruption is common in TBI and may occur almost instantaneously. ${ }^{108}$ Following a head injury, cerebrovascular endothelial cells often incur mechanical deformation, as well as shear stress injury, ${ }^{109}$ leading to increased tight junction permeability and leakage of molecules across the blood-brain barrier. Theoretically, this would lead to increased concentrations of CNS-enriched molecules in the blood and increased concentrations of blood-derived molecules in the CSF.

The best established clinical fluid biomarker for blood-brain barrier integrity is the $\mathrm{CSF} /$ serum albumin ratio (albumin produced by the liver has to cross the blood-brain barrier to reach the CSF). ${ }^{21}$ Some researchers caution against describing this measure as a bloodbrain barrier test and state that it actually reflects the blood-CSF barrier at the choroid plexus. ${ }^{110}$ However, in for example stroke, leaving the choroid plexus intact but injuring 
cerebrovascular endothelial cells, the CSF/serum albumin ratio may be increased, ${ }^{111}$ suggesting that $\mathrm{CSF} /$ serum albumin ratio probably is a marker of both barriers.

Increased CSF/serum albumin ratio has been reported in patients with severe TBI. ${ }^{112,113}$ In mild TBI, no such changes have been seen, ${ }^{74,114}$ suggesting that the blood-brain barrier remains rather intact or only opens up transiently in mild TBI, or that the CSF/serum albumin ratio is not a sensitive enough marker of blood-brain barrier injury in mild TBI.

It has been hypothesized that protein members of tight junctions could serve as plasma biomarkers for blood-brain barrier injury in TBI. ${ }^{115}$ One such protein is occludin, a $65-\mathrm{kDa}$ integral membrane protein that contributes to tight junction stabilization at barriers. ${ }^{115}$ However, this protein is not specific to the brain, but also expressed at high levels in testis, kidney, liver and lung. ${ }^{116}$ This may explain why increased plasma concentration of occludin has been observed, not only in concussed individuals, but also in patients with orthopaedic injury without concussion. ${ }^{117}$

\section{Neuroinflammation}

A large number of studies report an intrathecal acute phase inflammatory response following severe TBI, which is reflected in the CSF as increased concentrations of proinflammatory proteins such as IL-6, IL-8 and IL-10. ${ }^{112,113,118-128}$ Whether this reflects increased production of cytokines and interleukins within the CNS in response to the trauma, or leakage across a disrupted blood-brain barrier, or both, is unknown. The potential pathophysiological importance of the changes is supported by the association between a pro-inflammatory biomarker pattern and worse clinical outcome, not only in TBI, ${ }^{119,128}$ but also in other brain insults such as subarachnoid haemorrhage. ${ }^{129}$ 
In a pilot study including 16 paediatric patients with mild TBI and 20 paediatric patients who were scheduled for outpatient surgery but otherwise healthy, Berger et al. used multiplex bead technology to measure a large number of inflammatory proteins in serum. ${ }^{130}$ A combination of IL-6 and MMP-9 (increased serum concentrations of both in mild TBI) gave a sensitivity of $81 \%$ and specificity of $94 \%$ for diagnosing the condition. ${ }^{130}$ This is a result in need of replication given the low number of included subjects. More recently, blood concentrations of inflammatory molecules, such as substance P, soluble CD40 ligand, and tissue inhibitor of matrix metalloproteinases, have been associated with outcome in TBI patients. ${ }^{131}$

\section{Hypermetabolism, mitochondrial dysfunction and oxidative stress}

Cerebral energy perturbation appears to play a major role in TBI, which might be detectable using markers of mitochondrial dysfunction, oxidative stress and metabolic changes. In the context of mild TBI, the literature is scarce but a well-recognised feature of severe TBI is elevation of brain extracellular lactate concentrations and the lactate/pyruvate $(\mathrm{L} / \mathrm{P})$ ratio, which is associated with unfavourable clinical outcome. ${ }^{132}$ A recent pilot study comparing profiles of amino acids and other small molecules in serum samples from moderate to severe TBI patients identified a pattern of changes in the concentrations of nine metabolites, which could distinguish patients who developed post-TBI cognitive impairment from those who did not. ${ }^{133}$ Another pilot study showed 2-to 7-fold increases in plasma concentration of the antioxidant enzyme peroxiredoxin 6 hours to days following mild to severe TBI compared to healthy, non-concussed control individuals. How these changes relate to post-TBI changes in the CNS is currently unknown. The findings are also in need of replication. 


\section{Explorative approaches}

To identify novel biomarkers for TBI, more or less unbiased discovery approaches, such as proteomics, lipidomics, antigen panels to detect auto-antibodies, exosome analyses, and microRNA arrays, have been used. So far, most studies have been conducted in TBI models and the literature on human TBI is limited. In two independent explorative proteomics studies on human samples, serum amyloid A (SAA) protein was identified as a potential blood biomarker for TBI. ${ }^{134,135}$ However, animal model data suggests that this is due to TBIassociated up-regulation of SAA protein synthesis in the liver rather than the CNS injury per se. ${ }^{136}$ Other replicated findings from proteomics studies on TBI are currently lacking.

Using antigen panels, some studies suggest that TBI with blood-brain barrier disruption may induce the production of auto-antibodies against CNS antigens that may even be of pathophysiological relevance and potentially influence long-term outcome. ${ }^{137}$ Pilot studies suggest that this may occur even upon repetitive subconcussive head blows in American football players. ${ }^{138}$ Another hot topic is whether TBI may induce release of exosomes/microvesicles/microparticles across the blood-brain barrier into the bloodstream. Pilot data indicate that this may occur in severe TBI, ${ }^{139}$ but to the best of our knowledge data on mild TBI is lacking. Finally, lipidomics-derived data on brain-enriched sphingolipids suggest that these increase in plasma from stroke patients, ${ }^{140}$ which would be interesting to explore further in TBI.

Using microRNA arrays, elevated plasma levels of miR-16 and miR-92a in mild TBI were found, but the results are hard to interpret since severe TBI patients had lower levels and since the mild TBI levels were not different from those in orthopaedic injury patients. ${ }^{141}$ Starting with findings in a rat model of TBI, one study evaluated three significantly upregulated 
microRNAs (miR-93, miR-191, and miR-499) in sera from TBI patients. ${ }^{142}$ Serum miR-93, miR-191, and miR-499 levels were significantly increased in the TBI patients compared with controls at all examined time points, and associated with TBI severity and outcome, calling for additional validation studies of these potential markers.

\section{Biomarkers in PCS}

As discussed above, symptoms of mild TBI usually resolve within days to weeks, but in $15 \%$ of concussion patients neurological symptoms persist for months or more. ${ }^{9,10}$ The relationship between repetitive mild TBI and development of PCS is poorly understood, specifically to what degree such long-lasting symptoms are due to neuronal damage, and to what degree psychogenic or psychosocial mechanisms play a role. It is also important to point out that PCS symptoms are not brain-specific; they are often found also in non-TBI subjects. ${ }^{143}$ Standard blood-based biomarkers, such as S-100B, do not detect CNS injury in PCS but this may well be a sensitivity issue. ${ }^{144}$ In a recent pilot study of 16 patients with PCS and 15 matched healthy control individuals, it was shown that a subset of the PCS patients (those with symptoms for more than 1 year, $n=8$ ) had increased CSF concentrations of NF-L, which indicates ongoing axonal injury, and decreased CSF concentrations of the 42 amino acid form of the $A \beta$ (A $\beta 42)$, which may indicate $A \beta$ deposition in the brain parenchyma. ${ }^{145}$ These results warrant replication and also (if verified) long-term studies to assess whether this biomarker pattern may represent a prodrome of CTE.

\section{Chronic traumatic encephalopathy}

Chronic traumatic encephalopathy (CTE) is a neurodegenerative disease associated with repetitive head trauma. ${ }^{16}$ There are no established neuroimaging or fluid markers of CTE, ${ }^{146}$ 
although the gross morphological changes seen in advanced CTE may be visualized using standard neuroimaging techniques. ${ }^{147}$

In regards to in vivo markers of the molecular pathology in CTE, preliminary findings from positron emission tomography (PET) scanning using 2-(1-\{6-[(2-[fluorine18]fluoroethyl)(methyl)amino]-2-naphthyl\}-ethylidene)malononitrile (FDDNP), a ligand that binds to both neurofibrillary tangles and amyloid aggregates in the brain, of five retired players and five matched controls without a concussion history showed higher FDDNP signals in players compared with controls in all subcortical regions and the amygdala, which is a pattern distinct from that seen in Alzheimer's disease. ${ }^{148}$ Similar results were recently reported in a larger case series. ${ }^{149}$ Selective amyloid PET imaging in TBI has yielded ambiguous data, possibly due to the diffuse nature of trauma-related amyloid deposits that are less prone to bind amyloid ligands than the neuritic plaques seen in Alzheimer's disease. ${ }^{150}$ No data on fluid markers of tau and A $\beta$ pathology in CTE have been presented so far, although currently available CSF biomarkers for these two pathologies in Alzheimer's disease, along with CSF NF-L, may be regarded as promising candidates. Clinical biomarker studies with longitudinal follow-up of patients with chronic or progressive symptoms after TBI are warranted to learn more about the pathogenesis, risk factors and clinical course of CTE.

\section{Concluding remarks}

When reviewing the biomarker literature on mild TBI, PCS and CTE, it is clear that the field remains young; a lot of work remains to be done to optimize sample collection (including sampling time points) and storage, to identify and mitigate pre-analytical and analytical 
confounders and to optimize the employed assays to maximise their analytical sensitivity and specificity. Intense such work is ongoing in different research consortia.

Another issue with biomarker research in mild TBI is that there is currently no established reference (or "gold") standard validated against neuropathology to make the diagnosis. Further, the imaging methods are presently not sensitive enough to reliably detect mild TBI. We therefore have to rely on clinical criteria for mild TBI/concussion as the reference standard for making the diagnosis, which gives us rather heterogeneous groups to examine. This is an obvious limitation, but within such clinically diagnosed mild TBI groups, there may be individuals with more or less clear biomarker alterations. Longitudinal studies may then clarify whether mild TBI patients with more or less abnormal biomarker concentrations are more or less likely to develop PCS or related disorders. Predictive biomarker cut-points then have to be independently validated. Similarly, sample collections from patients with PCS, where there eventually may be autopsy data indicating CTE in some cases, will be useful to find biomarkers that predict CTE. All this will be iterative work, requiring extensive collaboration and sharing of materials, methods and samples.

In regards to blood-based biomarkers for TBI, promising data exist on the detection of acute and potentially also delayed effects of TBI, but more replication and validation work needs to be done and more biomarker candidates are warranted. It should be noted that brain pathology in mild TBI may be rather subtle, which puts extra demand on the analytical performance of the employed assays; targeted analyses of key molecules using ultrasensitive technologies will likely be important in this context. 
Several important issues in regards to the potential clinical usefulness of biomarkers for mild TBI could not be addressed in this review due to lack of data. For example, we have not been able to discuss what the optimal analytical platforms are (it is simply too early to make such claims), the potential of point-of-care testing, turnaround times in the emergency care unit and similar clinically highly relevant matters. The current state of this research field is that promising biomarker candidates exist but that the findings from individual studies, indicating potential clinical utility, now await independent replication. Once this requirement has been fulfilled and the clinical context of use for a certain biomarker has been established, the biomarker should be nominated for further standardisation work, including the development of certified reference materials and methods, to allow for proper assay standardisation, quality control and cut-point determination, and to optimise the technical aspects of the analytical methods to allow for rapid and cost-effective testing.

\section{Acknowledgements}

Work in the authors' laboratories is supported by the European Research Council, the Swedish Research Council, Swedish State Support for Clinical Research, the Torsten Söderberg Foundation, the Knut and Alice Wallenberg Foundation, VINNOVA and the Wolfson Foundation.

\section{Conflicts of interest}

Drs. Zetterberg and Blennow are listed as co-inventors on a US patent application for plasma tau as a brain injury marker and are co-founders of Brain Biomarker Solutions in Gothenburg AB, a GU Venture-based platform company at the University of Gothenburg. Dr. Blennow has served on advisory boards for Eli Lilly, Kyowa Kirin Pharma, Pfizer, and Roche. No other disclosures were reported. 


\section{References}

1. $\quad$ Easter, J.S., Haukoos, J.S., Meehan, W.P., Novack, V. \& Edlow, J.A. Will Neuroimaging Reveal a Severe Intracranial Injury in This Adult With Minor Head Trauma?: The Rational Clinical Examination Systematic Review. Jama 314, 26722681 (2015).

2. Teasdale, G. \& Jennett, B. Assessment of coma and impaired consciousness. A practical scale. Lancet 2, 81-84 (1974).

3. Sandsmark, D.K. Clinical Outcomes after Traumatic Brain Injury. Curr Neurol Neurosci Rep 16, 52 (2016).

4. Borg, J., et al. Diagnostic procedures in mild traumatic brain injury: results of the WHO Collaborating Centre Task Force on Mild Traumatic Brain Injury. J Rehabil Med, 61-75 (2004).

5. Levin, H.S. \& Diaz-Arrastia, R.R. Diagnosis, prognosis, and clinical management of mild traumatic brain injury. Lancet Neurol 14, 506-517 (2015).

6. McCrory, P., et al. Consensus statement on concussion in sport - the Third International Conference on Concussion in Sport held in Zurich, November 2008. Phys Sportsmed 37, 141-159 (2009).

7. Mu, W., Catenaccio, E. \& Lipton, M.L. Neuroimaging in Blast-Related Mild Traumatic Brain Injury. J Head Trauma Rehabil (2016).

8. Delouche, A., et al. Diffusion MRI: Pitfalls, literature review and future directions of research in mild traumatic brain injury. Eur J Radiol 85, 25-30 (2016).

9. Eisenberg, M.A., Andrea, J., Meehan, W. \& Mannix, R. Time interval between concussions and symptom duration. Pediatrics 132, 8-17 (2013).

10. Williams, W.H., Potter, S. \& Ryland, H. Mild traumatic brain injury and Postconcussion Syndrome: a neuropsychological perspective. J Neurol Neurosurg Psychiatry 81, 1116-1122 (2010).

11. Broshek, D.K., De Marco, A.P. \& Freeman, J.R. A review of post-concussion syndrome and psychological factors associated with concussion. Brain Inj 29, 228-237 (2015).

12. Ryan, L.M. \& Warden, D.L. Post concussion syndrome. Int Rev Psychiatry 15, 310 $316(2003)$.

13. Iverson, G.L. \& Lange, R.T. Examination of "postconcussion-like" symptoms in a healthy sample. Appl Neuropsychol 10, 137-144 (2003).

14. Rathbone, A.T., Tharmaradinam, S., Jiang, S., Rathbone, M.P. \& Kumbhare, D.A. A review of the neuro- and systemic inflammatory responses in post concussion symptoms: Introduction of the "post-inflammatory brain syndrome" PIBS. Brain Behav Immun 46, 1-16 (2015).

15. Corsellis, J.A., Bruton, C.J. \& Freeman-Browne, D. The aftermath of boxing. Psychol Med 3, 270-303 (1973).

16. Stein, T.D., Alvarez, V.E. \& McKee, A.C. Chronic traumatic encephalopathy: a spectrum of neuropathological changes following repetitive brain trauma in athletes and military personnel. Alzheimers Res Ther 6, 4 (2014).

17. DeKosky, S.T., Blennow, K., Ikonomovic, M.D. \& Gandy, S. Acute and chronic traumatic encephalopathies: pathogenesis and biomarkers. Nat Rev Neurol 9, 192-200 (2013).

18. Johanson, C.E., et al. Multiplicity of cerebrospinal fluid functions: New challenges in health and disease. Cerebrospinal Fluid Res 5, 10 (2008).

19. Louveau, A., et al. Structural and functional features of central nervous system lymphatic vessels. Nature 523, 337-341 (2015). 
20. Iliff, J.J., et al. A paravascular pathway facilitates CSF flow through the brain parenchyma and the clearance of interstitial solutes, including amyloid beta. Sci Transl Med 4, 147ra111 (2012).

21. Blennow, K., Hampel, H., Weiner, M. \& Zetterberg, H. Cerebrospinal fluid and plasma biomarkers in Alzheimer disease. Nat Rev Neurol 6, 131-144 (2010).

22. Duits, F.H., et al. Performance and complications of lumbar puncture in memory clinics: Results of the multicenter lumbar puncture feasibility study. Alzheimers Dement 12, 154-163 (2016).

23. Blennow, K. \& Nellgard, B. Amyloid beta 1-42 and tau in cerebrospinal fluid after severe traumatic brain injury. Neurology 62, 159; author reply 159-160 (2004).

24. Uhlen, M., et al. Proteomics. Tissue-based map of the human proteome. Science 347, 1260419 (2015).

25. Apweiler, R., et al. Approaching clinical proteomics: current state and future fields of application in fluid proteomics. Clin Chem Lab Med 47, 724-744 (2009).

26. Bolstad, N., Warren, D.J. \& Nustad, K. Heterophilic antibody interference in immunometric assays. Best Pract Res Clin Endocrinol Metab 27, 647-661 (2013).

27. Yoshimura, T., et al. Stability of pro-gastrin-releasing peptide in serum versus plasma. Tumour Biol 29, 224-230 (2008).

28. Plog, B.A., et al. Biomarkers of traumatic injury are transported from brain to blood via the glymphatic system. J Neurosci 35, 518-526 (2015).

29. Shi, M., et al. Salivary tau species are potential biomarkers of Alzheimer's disease. $J$ Alzheimers Dis 27, 299-305 (2011).

30. Devic, I., et al. Salivary alpha-synuclein and DJ-1: potential biomarkers for Parkinson's disease. Brain 134, e178 (2011).

31. Chen, A., et al. Multiplex analyte assays to characterize different dementias: brain inflammatory cytokines in poststroke and other dementias. Neurobiol Aging 38, 56-67 (2016).

32. Rissin, D.M., et al. Single-molecule enzyme-linked immunosorbent assay detects serum proteins at subfemtomolar concentrations. Nat Biotechnol 28, 595-599 (2010).

33. Todd, J., et al. Ultrasensitive flow-based immunoassays using single-molecule counting. Clin Chem 53, 1990-1995 (2007).

34. Lundberg, M., Eriksson, A., Tran, B., Assarsson, E. \& Fredriksson, S. Homogeneous antibody-based proximity extension assays provide sensitive and specific detection of low-abundant proteins in human blood. Nucleic Acids Res 39, e102 (2011).

35. Kricka, L.J. Human anti-animal antibody interferences in immunological assays. Clin Chem 45, 942-956 (1999).

36. Strathmann, F.G. \& Hoofnagle, A.N. Current and future applications of mass spectrometry to the clinical laboratory. Am J Clin Pathol 136, 609-616 (2011).

37. Sabbagh, B., Mindt, S., Neumaier, M. \& Findeisen, P. Clinical applications of MSbased protein quantification. Proteomics Clin Appl 10, 323-345 (2016).

38. Algattas, H. \& Huang, J.H. Traumatic Brain Injury pathophysiology and treatments: early, intermediate, and late phases post-injury. Int J Mol Sci 15, 309-341 (2014).

39. Schmechel, D., Marangos, P.J. \& Brightman, M. Neurone-specific enolase is a molecular marker for peripheral and central neuroendocrine cells. Nature 276, 834836 (1978).

40. Olsson, B., Zetterberg, H., Hampel, H. \& Blennow, K. Biomarker-based dissection of neurodegenerative diseases. Prog Neurobiol (2011).

41. Dash, P.K., Zhao, J., Hergenroeder, G. \& Moore, A.N. Biomarkers for the diagnosis, prognosis, and evaluation of treatment efficacy for traumatic brain injury.

Neurotherapeutics 7, 100-114 (2010). 
42. Ramont, L., et al. Effects of hemolysis and storage condition on neuron-specific enolase (NSE) in cerebrospinal fluid and serum: implications in clinical practice. Clin Chem Lab Med 43, 1215-1217 (2005).

43. Bohmer, A.E., et al. Neuron-specific enolase, S100B, and glial fibrillary acidic protein levels as outcome predictors in patients with severe traumatic brain injury. Neurosurgery 68, 1624-1630; discussion 1630-1621 (2011).

44. Chiaretti, A., et al. NGF, DCX, and NSE upregulation correlates with severity and outcome of head trauma in children. Neurology 72, 609-616 (2009).

45. Varma, S., et al. F2-isoprostane and neuron-specific enolase in cerebrospinal fluid after severe traumatic brain injury in infants and children. $J$ Neurotrauma 20, 781-786 (2003).

46. Berger, R.P., et al. Neuron-specific enolase and S100B in cerebrospinal fluid after severe traumatic brain injury in infants and children. Pediatrics 109, E31 (2002).

47. Ross, S.A., Cunningham, R.T., Johnston, C.F. \& Rowlands, B.J. Neuron-specific enolase as an aid to outcome prediction in head injury. Br J Neurosurg 10, 471-476 (1996).

48. Pelsers, M.M., et al. Brain- and heart-type fatty acid-binding proteins in the brain: tissue distribution and clinical utility. Clin Chem 50, 1568-1575 (2004).

49. Skogseid, I.M., Nordby, H.K., Urdal, P., Paus, E. \& Lilleaas, F. Increased serum creatine kinase BB and neuron specific enolase following head injury indicates brain damage. Acta Neurochir (Wien) 115, 106-111 (1992).

50. de Kruijk, J.R., Leffers, P., Menheere, P.P., Meerhoff, S. \& Twijnstra, A. S-100B and neuron-specific enolase in serum of mild traumatic brain injury patients. A comparison with health controls. Acta Neurol Scand 103, 175-179 (2001).

51. Wolf, H., et al. Predictive value of neuromarkers supported by a set of clinical criteria in patients with mild traumatic brain injury: S100B protein and neuron-specific enolase on trial: clinical article. J Neurosurg 118, 1298-1303 (2013).

52. Shahim, P., et al. Blood biomarkers for brain injury in concussed professional ice hockey players. JAMA Neurol 71, 684-692 (2014).

53. Chen, F., Sugiura, Y., Myers, K.G., Liu, Y. \& Lin, W. Ubiquitin carboxyl-terminal hydrolase L1 is required for maintaining the structure and function of the neuromuscular junction. Proc Natl Acad Sci U S A 107, 1636-1641 (2010).

54. Thompson, R.J., Doran, J.F., Jackson, P., Dhillon, A.P. \& Rode, J. PGP 9.5--a new marker for vertebrate neurons and neuroendocrine cells. Brain Res 278, 224-228 (1983).

55. Takami, Y., et al. Ubiquitin carboxyl-terminal hydrolase L1, a novel deubiquitinating enzyme in the vasculature, attenuates NF-kappaB activation. Arterioscler Thromb Vasc Biol 27, 2184-2190 (2007).

56. Campbell, L.K., Thomas, J.R., Lamps, L.W., Smoller, B.R. \& Folpe, A.L. Protein gene product 9.5 (PGP 9.5) is not a specific marker of neural and nerve sheath tumors: an immunohistochemical study of 95 mesenchymal neoplasms. Mod Pathol 16, 963 969 (2003).

57. Brophy, G.M., et al. Biokinetic analysis of ubiquitin C-terminal hydrolase-L1 (UCHL1) in severe traumatic brain injury patient biofluids. J Neurotrauma 28, 861-870 (2011).

58. Kulbe, J.R. \& Geddes, J.W. Current status of fluid biomarkers in mild traumatic brain injury. Exp Neurol 275 Pt 3, 334-352 (2016).

59. Papa, L., et al. Serum levels of ubiquitin C-terminal hydrolase distinguish mild traumatic brain injury from trauma controls and are elevated in mild and moderate 
traumatic brain injury patients with intracranial lesions and neurosurgical intervention. J Trauma Acute Care Surg 72, 1335-1344 (2012).

60. Diaz-Arrastia, R., et al. Acute biomarkers of traumatic brain injury: relationship between plasma levels of ubiquitin C-terminal hydrolase-L1 and glial fibrillary acidic protein. J Neurotrauma 31, 19-25 (2014).

61. Kaplan, G.B., Vasterling, J.J. \& Vedak, P.C. Brain-derived neurotrophic factor in traumatic brain injury, post-traumatic stress disorder, and their comorbid conditions: role in pathogenesis and treatment. Behav Pharmacol 21, 427-437 (2010).

62. Korley, F.K., et al. Circulating Brain-Derived Neurotrophic Factor Has Diagnostic and Prognostic Value in Traumatic Brain Injury. J Neurotrauma 33, 215-225 (2016).

63. Simon, D., Nascimento, R.I., Filho, E.M., Bencke, J. \& Regner, A. Plasma brainderived neurotrophic factor levels after severe traumatic brain injury. Brain Inj 30, 2328 (2016).

64. Zetterberg, H., Smith, D.H. \& Blennow, K. Biomarkers of mild traumatic brain injury in cerebrospinal fluid and blood. Nat Rev Neurol 9, 201-210 (2013).

65. Morris, M., Maeda, S., Vossel, K. \& Mucke, L. The many faces of tau. Neuron 70, 410-426 (2011).

66. Goedert, M., Spillantini, M.G. \& Crowther, R.A. Cloning of a big tau microtubuleassociated protein characteristic of the peripheral nervous system. Proc Natl Acad Sci U S A 89, 1983-1987 (1992).

67. Trojanowski, J.Q., Schuck, T., Schmidt, M.L. \& Lee, V.M. Distribution of tau proteins in the normal human central and peripheral nervous system. $J$ Histochem Cytochem 37, 209-215 (1989).

68. Friede, R.L. \& Samorajski, T. Axon caliber related to neurofilaments and microtubules in sciatic nerve fibers of rats and mice. Anat Rec 167, 379-387 (1970).

69. Ost, M., et al. Initial CSF total tau correlates with 1-year outcome in patients with traumatic brain injury. Neurology 67, 1600-1604 (2006).

70. Franz, G., et al. Amyloid beta 1-42 and tau in cerebrospinal fluid after severe traumatic brain injury. Neurology 60, 1457-1461 (2003).

71. Zemlan, F.P., et al. C-tau biomarker of neuronal damage in severe brain injured patients: association with elevated intracranial pressure and clinical outcome. Brain Res 947, 131-139 (2002).

72. Hesse, C., et al. Transient increase in total tau but not phospho-tau in human cerebrospinal fluid after acute stroke. Neurosci Lett 297, 187-190 (2001).

73. Neselius, S., et al. CSF-biomarkers in Olympic boxing: diagnosis and effects of repetitive head trauma. PLOS ONE 7, e33606 (2012).

74. Zetterberg, H., et al. Neurochemical aftermath of amateur boxing. Arch Neurol 63, 1277-1280 (2006).

75. Neselius, S., Zetterberg, H., Blennow, K., Marcusson, J. \& Brisby, H. Increased CSF levels of phosphorylated neurofilament heavy protein following bout in amateur boxers. PLoS ONE 8, e81249 (2013).

76. Guzel, A., Karasalihoglu, S., Aylanc, H., Temizoz, O. \& Hicdonmez, T. Validity of serum tau protein levels in pediatric patients with minor head trauma. Am J Emerg Med 28, 399-403 (2010).

77. Bulut, M., et al. Tau protein as a serum marker of brain damage in mild traumatic brain injury: preliminary results. Adv Ther 23, 12-22 (2006).

78. Kavalci, C., et al. The value of serum tau protein for the diagnosis of intracranial injury in minor head trauma. Am J Emerg Med 25, 391-395 (2007). 
79. Al Nimer, F., et al. Comparative Assessment of the Prognostic Value of Biomarkers in Traumatic Brain Injury Reveals an Independent Role for Serum Levels of Neurofilament Light. PLOS ONE 10, e0132177 (2015).

80. Randall, J., et al. Tau proteins in serum predict neurological outcome after hypoxic brain injury from cardiac arrest: results of a pilot study. Resuscitation 84, 351-356 (2013).

81. Kuhle, J., et al. Comparison of three analytical platforms for quantification of the neurofilament light chain in blood samples: ELISA, electrochemiluminescence immunoassay and Simoa. Clin Chem Lab Med (2016).

82. Zetterberg, H., et al. Plasma tau levels in Alzheimer's disease. Alzheimers Res Ther 5, 9 (2013).

83. Bogoslovsky, T., et al. Increases of Plasma Levels of Glial Fibrillary Acidic Protein, Tau, and Amyloid beta up to 90 Days after Traumatic Brain Injury. J Neurotrauma (2016).

84. Olivera, A., et al. Peripheral Total Tau in Military Personnel Who Sustain Traumatic Brain Injuries During Deployment. JAMA Neurol 72, 1109-1116 (2015).

85. Shahim, P., et al. Serum Tau Fragments Predict Return to Play in Concussed Professional Ice Hockey Players. J Neurotrauma (2016).

86. Bazarian, J.J., Zemlan, F.P., Mookerjee, S. \& Stigbrand, T. Serum S-100B and cleaved-tau are poor predictors of long-term outcome after mild traumatic brain injury. Brain Inj 20, 759-765 (2006).

87. Ma, M., Lindsell, C.J., Rosenberry, C.M., Shaw, G.J. \& Zemlan, F.P. Serum cleaved tau does not predict postconcussion syndrome after mild traumatic brain injury. Am J Emerg Med 26, 763-768 (2008).

88. Gisslen, M., et al. Plasma Concentration of the Neurofilament Light Protein (NFL) is a Biomarker of CNS Injury in HIV Infection: A Cross-Sectional Study. EBioMedicine 3, 135-140 (2016).

89. Oliver, J., et al. Serum Neurofilament Light in American Football Athletes Over the Course of a Season. J Neurotrauma (2015).

90. Gatson, J.W., et al. Detection of neurofilament-H in serum as a diagnostic tool to predict injury severity in patients who have suffered mild traumatic brain injury. $J$ Neurosurg 121, 1232-1238 (2014).

91. Pike, B.R., et al. Accumulation of non-erythroid alpha II-spectrin and calpain-cleaved alpha II-spectrin breakdown products in cerebrospinal fluid after traumatic brain injury in rats. J Neurochem 78, 1297-1306 (2001).

92. Pineda, J.A., et al. Clinical significance of alphaII-spectrin breakdown products in cerebrospinal fluid after severe traumatic brain injury. $J$ Neurotrauma 24, 354-366 (2007).

93. Farkas, O., et al. Spectrin breakdown products in the cerebrospinal fluid in severe head injury--preliminary observations. Acta Neurochir (Wien) 147, 855-861 (2005).

94. Mondello, S., et al. alphaII-spectrin breakdown products (SBDPs): diagnosis and outcome in severe traumatic brain injury patients. $J$ Neurotrauma 27, 1203-1213 (2010).

95. Czeiter, E., et al. Brain injury biomarkers may improve the predictive power of the IMPACT outcome calculator. J Neurotrauma 29, 1770-1778 (2012).

96. Siman, R., et al. A panel of neuron-enriched proteins as markers for traumatic brain injury in humans. J Neurotrauma 26, 1867-1877 (2009).

97. Siman, R., et al. Evidence That the Blood Biomarker SNTF Predicts Brain Imaging Changes and Persistent Cognitive Dysfunction in Mild TBI Patients. Front Neurol 4, 190 (2013). 
98. Siman, R., et al. Serum SNTF Increases in Concussed Professional Ice Hockey Players and Relates to the Severity of Post-Concussion Symptoms. J Neurotrauma (2014).

99. Barbarese, E., et al. Expression and localization of myelin basic protein in oligodendrocytes and transfected fibroblasts. J Neurochem 51, 1737-1745 (1988).

100. Cerri, C.G., Silani, V. \& Scarlato, G. Oligoclonal immunoglobulins and immunoreactive myelin basic protein in the cerebrospinal fluid of patients with multiple sclerosis and other neurological diseases. Acta Neurol (Napoli) 7, 311-314 (1985).

101. Su, E., et al. Increased CSF concentrations of myelin basic protein after TBI in infants and children: absence of significant effect of therapeutic hypothermia. Neurocrit Care 17, 401-407 (2012).

102. Berger, R.P., et al. Serum neuron-specific enolase, S100B, and myelin basic protein concentrations after inflicted and noninflicted traumatic brain injury in children. $J$ Neurosurg 103, 61-68 (2005).

103. Yan, E.B., et al. Post-traumatic hypoxia is associated with prolonged cerebral cytokine production, higher serum biomarker levels, and poor outcome in patients with severe traumatic brain injury. J Neurotrauma 31, 618-629 (2014).

104. Unden, J., Ingebrigtsen, T. \& Romner, B. Scandinavian guidelines for initial management of minimal, mild and moderate head injuries in adults: an evidence and consensus-based update. BMC Med 11, 50 (2013).

105. McMahon, P.J., et al. Measurement of the GFAP-BDP Biomarker for the Detection of Traumatic Brain Injury Compared to CT and MRI. J Neurotrauma (2014).

106. Papa, L., et al. GFAP out-performs S100beta in detecting traumatic intracranial lesions on computed tomography in trauma patients with mild traumatic brain injury and those with extracranial lesions. J Neurotrauma 31, 1815-1822 (2014).

107. Korfias, S., et al. Slight and short-lasting increase of serum S-100B protein in extracranial trauma. Brain Inj 20, 867-872 (2006).

108. Hay, J.R., Johnson, V.E., Young, A.M., Smith, D.H. \& Stewart, W. Blood-Brain Barrier Disruption Is an Early Event That May Persist for Many Years After Traumatic Brain Injury in Humans. J Neuropathol Exp Neurol 74, 1147-1157 (2015).

109. Rodriguez-Baeza, A., Reina-de la Torre, F., Poca, A., Marti, M. \& Garnacho, A. Morphological features in human cortical brain microvessels after head injury: a threedimensional and immunocytochemical study. Anat Rec A Discov Mol Cell Evol Biol 273, 583-593 (2003).

110. Reiber, H. \& Peter, J.B. Cerebrospinal fluid analysis: disease-related data patterns and evaluation programs. J Neurol Sci 184, 101-122 (2001).

111. Brouns, R., Wauters, A., De Surgeloose, D., Marien, P. \& De Deyn, P.P. Biochemical markers for blood-brain barrier dysfunction in acute ischemic stroke correlate with evolution and outcome. Eur Neurol 65, 23-31 (2011).

112. Csuka, E., et al. IL-10 levels in cerebrospinal fluid and serum of patients with severe traumatic brain injury: relationship to IL-6, TNF-alpha, TGF-beta1 and blood-brain barrier function. J Neuroimmunol 101, 211-221 (1999).

113. Kossmann, T., et al. Intrathecal and serum interleukin- 6 and the acute-phase response in patients with severe traumatic brain injuries. Shock 4, 311-317 (1995).

114. Blennow, K., et al. No neurochemical evidence of brain injury after blast overpressure by repeated explosions or firing heavy weapons. Acta Neurol Scand 123, 245-251 (2011).

115. Cummins, P.M. Occludin: one protein, many forms. Mol Cell Biol 32, 242-250 (2012). 
116. Saitou, M., et al. Mammalian occludin in epithelial cells: its expression and subcellular distribution. Eur J Cell Biol 73, 222-231 (1997).

117. Shan, R., et al. A New Panel of Blood Biomarkers for the Diagnosis of Mild Traumatic Brain Injury/Concussion in Adults. J Neurotrauma 33, 49-57 (2016).

118. Semple, B.D., Bye, N., Rancan, M., Ziebell, J.M. \& Morganti-Kossmann, M.C. Role of CCL2 (MCP-1) in traumatic brain injury (TBI): evidence from severe TBI patients and CCL2-/- mice. J Cereb Blood Flow Metab 30, 769-782 (2010).

119. Kirchhoff, C., et al. Cerebrospinal IL-10 concentration is elevated in non-survivors as compared to survivors after severe traumatic brain injury. Eur J Med Res 13, 464-468 (2008).

120. Goodman, J.C., Van, M., Gopinath, S.P. \& Robertson, C.S. Pro-inflammatory and proapoptotic elements of the neuroinflammatory response are activated in traumatic brain injury. Acta Neurochir Suppl 102, 437-439 (2008).

121. Buttram, S.D., et al. Multiplex assessment of cytokine and chemokine levels in cerebrospinal fluid following severe pediatric traumatic brain injury: effects of moderate hypothermia. J Neurotrauma 24, 1707-1717 (2007).

122. Phillips, D.J., et al. Activin a release into cerebrospinal fluid in a subset of patients with severe traumatic brain injury. J Neurotrauma 23, 1283-1294 (2006).

123. Maier, B., et al. Delayed elevation of soluble tumor necrosis factor receptors $\mathrm{p} 75$ and p55 in cerebrospinal fluid and plasma after traumatic brain injury. Shock 26, 122-127 (2006).

124. Shiozaki, T., et al. Cerebrospinal fluid concentrations of anti-inflammatory mediators in early-phase severe traumatic brain injury. Shock 23, 406-410 (2005).

125. Singhal, A., et al. Association between cerebrospinal fluid interleukin-6 concentrations and outcome after severe human traumatic brain injury. $J$ Neurotrauma 19, 929-937 (2002).

126. Stahel, P.F., et al. Intrathecal levels of complement-derived soluble membrane attack complex (sC5b-9) correlate with blood-brain barrier dysfunction in patients with traumatic brain injury. J Neurotrauma 18, 773-781 (2001).

127. Bell, M.J., et al. Interleukin-6 and interleukin-10 in cerebrospinal fluid after severe traumatic brain injury in children. $J$ Neurotrauma 14, 451-457 (1997).

128. Kumar, R.G., et al. Acute CSF interleukin-6 trajectories after TBI: associations with neuroinflammation, polytrauma, and outcome. Brain Behav Immun 45, 253-262 (2015).

129. Csajbok, L.Z., Nylen, K., Ost, M., Sonander, H. \& Nellgard, B. In-hospital C-reactive protein predicts outcome after aneurysmal subarachnoid haemorrhage treated by endovascular coiling. Acta Anaesthesiol Scand 59, 255-264 (2015).

130. Berger, R.P., Ta'asan, S., Rand, A., Lokshin, A. \& Kochanek, P. Multiplex assessment of serum biomarker concentrations in well-appearing children with inflicted traumatic brain injury. Pediatr Res 65, 97-102 (2009).

131. Lorente, L. New Prognostic Biomarkers in Patients With Traumatic Brain Injury. Arch Trauma Res 4, e30165 (2015).

132. Timofeev, I., et al. Cerebral extracellular chemistry and outcome following traumatic brain injury: a microdialysis study of 223 patients. Brain 134, 484-494 (2011).

133. Yi, L., et al. Serum Metabolic Profiling Reveals Altered Metabolic Pathways in Patients with Post-traumatic Cognitive Impairments. Sci Rep 6, 21320 (2016).

134. Hergenroeder, G., et al. Identification of serum biomarkers in brain-injured adults: potential for predicting elevated intracranial pressure. $J$ Neurotrauma 25, 79-93 (2008). 
135. Gao, W., Lu, C., Kochanek, P.M. \& Berger, R.P. Serum amyloid A is increased in children with abusive head trauma: a gel-based proteomic analysis. Pediatr Res 76, 280-286 (2014).

136. Villapol, S., et al. Hepatic expression of serum amyloid A1 is induced by traumatic brain injury and modulated by telmisartan. Am J Pathol 185, 2641-2652 (2015).

137. Raad, M., et al. Auto-antibodies in traumatic brain injury and central nervous system trauma. Neuroscience 281, 16-23 (2014).

138. Marchi, N., et al. Consequences of repeated blood-brain barrier disruption in football players. PLoS ONE 8, e56805 (2013).

139. Nekludov, M., Mobarrez, F., Gryth, D., Bellander, B.M. \& Wallen, H. Formation of microparticles in the injured brain of patients with severe isolated traumatic brain injury. J Neurotrauma 31, 1927-1933 (2014).

140. Sheth, S.A., Iavarone, A.T., Liebeskind, D.S., Won, S.J. \& Swanson, R.A. Targeted Lipid Profiling Discovers Plasma Biomarkers of Acute Brain Injury. PLoS ONE 10, e0129735 (2015).

141. Redell, J.B., Moore, A.N., Ward, N.H., 3rd, Hergenroeder, G.W. \& Dash, P.K. Human traumatic brain injury alters plasma microRNA levels. $J$ Neurotrauma 27, 2147-2156 (2010).

142. Yang, T., et al. Elevated serum miR-93, miR-191, and miR-499 are noninvasive biomarkers for the presence and progression of traumatic brain injury. J Neurochem 137, 122-129 (2016).

143. Losoi, H., et al. Recovery from Mild Traumatic Brain Injury in Previously Healthy Adults. J Neurotrauma 33, 766-776 (2016).

144. Babcock, L., Byczkowski, T., Wade, S.L., Ho, M. \& Bazarian, J.J. Inability of S100B to predict postconcussion syndrome in children who present to the emergency department with mild traumatic brain injury: a brief report. Pediatr Emerg Care 29, 458-461 (2013).

145. Shahim, P., et al. Neurochemical aftermath of repetitive mild traumatic brain injury JAMA Neurol (2016).

146. Sundman, M., Doraiswamy, P.M. \& Morey, R.A. Neuroimaging assessment of early and late neurobiological sequelae of traumatic brain injury: implications for CTE. Front Neurosci 9, 334 (2015).

147. Corsellis, J.A. Boxing and the brain. Bmj 298, 105-109 (1989).

148. Small, G.W., et al. PET scanning of brain tau in retired national football league players: preliminary findings. Am J Geriatr Psychiatry 21, 138-144 (2013).

149. Barrio, J.R., et al. In vivo characterization of chronic traumatic encephalopathy using [F-18]FDDNP PET brain imaging. Proc Natl Acad Sci U S A 112, E2039-2047 (2015).

150. Kawai, N., et al. Detection of brain amyloid beta deposition in patients with neuropsychological impairment after traumatic brain injury: PET evaluation using Pittsburgh Compound-B. Brain Inj 27, 1026-1031 (2013). 
Box 1: Mild traumatic brain injury and related conditions

\begin{tabular}{|l|l|}
\hline Mild traumatic brain injury (concussion) & $\begin{array}{l}\text { Rapid-onset neuronal dysfunction caused by } \\
\text { an external mechanical force to the brain } \\
\text { without detectable gross pathology, such as } \\
\text { haemorrhage }\end{array}$ \\
\hline Post-concussive syndrome & $\begin{array}{l}\text { A complex disorder in which various } \\
\text { symptoms, such as headache and dizziness, } \\
\text { last for weeks to months after concussion }\end{array}$ \\
\hline Chronic traumatic encephalopathy & $\begin{array}{l}\text { A progressive tauopathy mainly found in } \\
\text { people with a history of repetitive head } \\
\text { injuries }\end{array}$ \\
\hline
\end{tabular}


Figure 1: Candidate fluid biomarkers for pathophysiological processes related to traumatic brain injury (TBI). Tau and neurofilament light (NF-L) and heavy (NF-H), as well as spectrin breakdown products (SBDPs), are biomarkers for axonal injury. Neuron-specific enolase (NSE) and ubiquitin C-terminal hydrolase-L1 (UCHL1) are proteins enriched in the neuronal soma and putative biomarkers for neuronal injury. Activated astrocytes and microglia in TBI secrete S-100B and glial fibrillary acidic protein (GFAP), as well as interleukins, cytokines and matrix metalloproteinases. $\mathrm{CSF} /$ serum albumin ratio is an established biomarker for blood-brain and/or blood-CSF barrier integrity. Plasma occludin is a candidate biomarker in the blood for the same function.

Figure 2: Hypothetical model of temporal brain changes in traumatic brain injury (TBI) not complicated by secondary insults. Blood-brain barrier disruption and axonal disconnection are thought to occur more or less instantaneously, probably together with fast astrocytic and microglial activation and induction of a hypermetabolic state that may induce long-term oxidative stress and mitochondrial dysfunction. Microglial activation may also linger and take part in a more delayed response with proper neuroinflammation. Wallerian degeneration may go on for months and even years following the injury and is signified by breakdown of axonal debris distal to the injury. The optimal time point for biomarker sampling will depend on what aspect of the TBI process the biomarker candidate is thought to reflect; this has to be carefully evaluated for each biomarker candidate in animal models and in human studies with repeated sampling over time. 